\title{
Multi-model Simulations of Chicken Limb Morphogenesis
}

\author{
R. Chaturvedi ${ }^{1}$, J.A. Izaguirre ${ }^{1}$, C. Huang ${ }^{1}$, T. Cickovski ${ }^{1}$, P. Virtue ${ }^{1}$, \\ G. Thomas ${ }^{2}$, G. Forgacs ${ }^{3}$, M. Alber ${ }^{4}$, G. Hentschel ${ }^{5}$, S.A. Newman ${ }^{6}$, and J.A. Glazier ${ }^{7}$ \\ ${ }^{1}$ Department of Computer Science and Engineering, University of Notre Dame, IN 46556 \\ Corresponding author: izaguirr@nd.edu \\ Tel: (574) 631-7454 Fax: (574) 727-0873 \\ ${ }^{2}$ Department of Physics, University of Notre Dame, Notre Dame, IN 46556 \\ ${ }^{3}$ Departments of Physics and Biology, University of Missouri, Columbia, MO 65201 \\ ${ }^{4}$ Department of Mathematics, University of Notre Dame, Notre Dame, IN 46556 \\ ${ }^{5}$ Department of Physics, Emory University, Atlanta, GA 30332 \\ ${ }^{6}$ Department of Cell Biology and Anatomy, New York Medical College, \\ Valhalla, NY10595 \\ ${ }^{7}$ Biocomplexity Institute and Department of Physics, Indiana University, \\ Bloomington, IN 47405
}

\begin{abstract}
Early development of multicellular organisms (morphogenesis) is a complex phenomenon. We present COMPUCELL, a multi-model software framework for simulations of morphogenesis. As an example, we simulate the formation of the skeletal pattern in the avian limb bud, which requires simulations based on interactions of the genetic regulatory network with generic cellular mechanisms (cell adhesion, haptotaxis, and chemotaxis). A combination of a rule-based state automaton and sets of differential equations, both subcellular ordinary differential equations (ODEs) and domain-level reaction-diffusion partial differential equations (PDEs) models genetic regulation. This regulation controls the differentiation of cells, and also cell-cell and cell-extracellular matrix interactions that give rise to cell pattern formation and cell rearrangements such as mesenchymal condensation. The cellular Potts model (CPM) models cell dynamics (cell movement and rearrangement). These models couple; COMPUCELL provides an integrated framework for such computations. Binaries for Microsoft Windows and Solaris are available ${ }^{1}$. Source code is available on request, via email: compucell@cse.nd.edu.
\end{abstract}

\section{Introduction}

In the fields of bioinformatics and computational biology an aim is to link the wealth of data (e.g. genetic sequences and genetic regulatory networks) to the understanding of biological processes such as development of multicellular organisms (morphogenesis). Computational systems biology concerns itself with creating such integrated models. Relatively little integrated modeling of multicellular organisms exists; examples are: development models of the Drosophila embryo [1], and analysis of the gene

\footnotetext{
${ }^{1}$ http: //www. nd. edu/ lcls/compucell
} 
regulatory network of developing sea urchin [2]. These efforts are confined mainly to the modeling of gene regulation. For morphogenesis, we need to model cell behaviors like release and absorption of diffusible factors, adhesion, and motility [3], in addition to differential regulation of gene activity.

We have implemented the software framework COMPUCELL to model early morphogenesis. It allows interaction of the genetic regulatory network with cellular mechanisms; features include biosynthesis, cell adhesion, haptotaxis (the movement of cells along a gradient of a molecule deposited on a substrate) and chemotaxis (the movement of cells along a gradient of a chemical diffusing in the extracellular environment), and diffusion of morphogens (molecules released by cells that affect the behavior of other cells during development). The interplay of these factors results in arrangements of cells specific to a given organism, cf. [4]. We describe the model and apply it to simulate the skeletal pattern formation in avian (chicken) limb.

Biological Background: Skeletal pattern formation occurs within a mesenchymal tissue (mesenchyme are cells arranged loosely in a hydrated extracellular matrix (ECM); they make only minimal contact with one another) surrounded by a thin bounding layer, the ectoderm; Figure 1 shows a schematic of vertebrate limb. Our two-dimensional (2-d) simulation attempts to reproduce the pattern of development of bones in the forelimb of a chicken viewed from above when it lies palm down on a flat surface. The long axis from the body to the digits is the proximodistal direction (proximal means close to the body); from the thumb to the little finger is the anteroposterior direction. Thickness of the limb, from back to front (the dorsoventral direction) is one skeletal element throughout, justifying our 2-d simplification. Although asymmetry along the dorsoventral axis as seen in the typical paddle shape of limb is important to function, our initial simulations ignore it in favor of the more striking asymmetries along the two other axes. Apical ectodermal ridge (AER), the narrow strip of the ectoderm running along the apex of the growing limb bud, is necessary for elongation and patterning of limb. AER releases fibroblast growth factors (FGFs), which control mitosis (division) of mesenchymal cells. FGF concentrations increase in the distal direction. Rates of cell division are known experimentally for the avian limb [5]. Limb growth and pattern formation has been conceptualized by considering the space within the developing limb to comprise three zones - (1) apical zone where only growth takes place, (2) active zone where dynamics of pattern formation and cell rearrangement into precartilage condensations occur and (3) frozen zone where condensations have progressed to differentiated cartilage and no additional patterning occurs. In development in species with a bony skeleton, bone later isomorphically replaces the cartilaginous skeleton. Active zone may be the locus of a reactiondiffusion system in which one or more members of the TGF- $\beta$ family of growth factors act as the activating morphogens [6]. Thus the active zone (2) is further classified into (2a) cell active zone where cells rearrange locally into precartilage condensations, (2b) reaction-diffusion active zone where reaction-diffusion mechanisms operate. Growth continues in both the active zones but not in the frozen zone. At sites of incipient condensation, cells bearing a second FGF receptor may release a laterally inhibitory morphogen, a necessary component of some pattern-forming reactiondiffusion schemes. Rather than use a reaction-diffusion scheme based on the actual 
biochemical interactions (they are still poorly characterized) we choose for heuristic purposes to use the well-studied Schnakenberg reaction-diffusion equations, Equation (5). We refer to ' $u$ ' in Schnakenberg equations as the reaction-diffusion activator, rather than identify it with a specific molecule TGF- $\beta$.

Cells sensing activator levels above a threshold secrete an adhesive material onto the substrate and become intrinsically stickier than surrounding cells. In the actual limb, TGF- $\beta$ induces cells in active zones to produce the ECM glycoprotein fibronectin [7]. Fibronectin adheres to the cell surface, causing cells to accumulate at focal sites. Cells at these sites also become more adhesive to one another by producing a homophilic cell-surface adhesion protein N-cadherin [8]. The spatiotemporal pattern of activator thus results in a corresponding set of cell condensations. In the description of our model and simulation results for avian limb, we refer to the secreted substrate adhesive molecule that promotes haptotaxis as SAM (surface adhesion molecule) and the cell-cell adhesive molecule as CAM (cell adhesion molecule). The current model omits the mitogen (FGF) field due to AER, instead assuming that cell division is uniform throughout the limb bud [5].

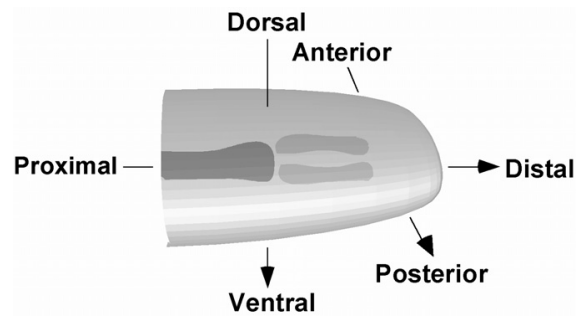

Fig. 1. Schematic representation of a developing vertebrate limb: We indicate the three major axes and the first two tiers of skeletal elements to form. In the chicken forelimb these elements are the humerus, shown as already differentiated (dark gray), followed by the radius and ulna, which are in the process of forming (light gray). Still to form are the wrist bones and digits. The apical ectodermal ridge runs along the distal tip of the limb approximately between the two points that the arrow indicating the anteroposterior axis intersects.

Brief Description of the Model: Steinberg's Differential Adhesion Hypothesis $(D A H)$ uses cell-cell and cell-matrix adhesive interactions to explain cell rearrangement, including mesenchymal cell condensation [9]. This generic aspect of cell behavior is modeled using CPM [10], which we have extended to account for haptotaxis and chemotaxis. Cells may respond to morphogens they or their neighbors produce by altering their gene activity in continuous or discontinuous (switch-like) fashion; such nonlinear feedback loops may lead to differentiation into more specific cell types. We model the network of expressed genes and their products as a set of rules that trigger growth, cell division, cell death, secretion of morphogens and strength of adhesion between cells; in the limb they are mediated by level of activity and distribution of growth factors. [11] reviews these and other approaches to modeling gene regulatory networks. A reaction-diffusion mechanism may underlie limb skeletal pattern formation as well as other biological spatiotemporal patterning [12-14]. We propose a 
model that uses domain-level PDEs to simulate reaction-diffusion mechanisms. In our biological example, these PDEs generate morphogen concentration distributions that establish a prepattern for mesenchymal cell condensation. The rules also interact with the stochastic CPM which governs dynamics and geometry of cell growth and division. Domain growth follows from cell growth. Details are provided below.

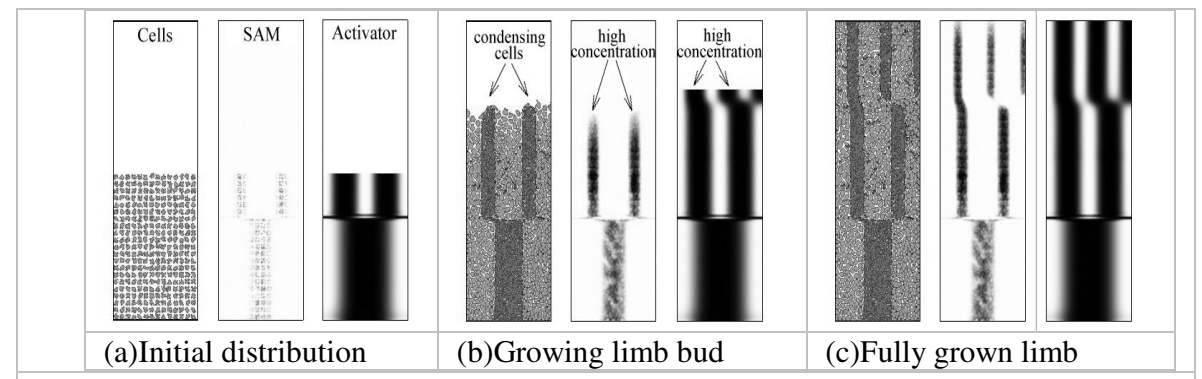

Fig. 2. Simulation of skeletal pattern formation in avian limb using COMPUCELL. For full limb, height to width ratio is 3 to 1 . Figure not to scale.

Simulation of Avian Limb Development Using CoMPUCELL: Figure 2 presents COMPUCELL simulation results of the biological model: (i) The pre-pattern formed by the morphogen (activator) chemical field (middle frame), (ii) The SAM concentration field produced by the cells in response to the activator after a delay (right frame), and (iii) The organization of mesenchymal cells into precartilaginous tissue in response to these fields (left frame). The typical bone pattern (Figure 1) of one proximal rod-shaped element followed by two elements of the mid-arm, and then three digits reflects the temporal progress of chicken limb morphogenesis. It is generally similar to formation of other vertebrate limbs. Our long-term goal is to explore developmental mechanisms such as avian limb pattern formation using realistic gene regulatory/reaction-diffusion networks and cell-cell adhesion measurements. In the absence of information on the interactions and quantities required for a biologically accurate simulation, the current simulations restrict themselves to describing the major biological issues involved in avian limb bud development. We have focused on simulating mesenchymal cell condensation and emergence of a skeletal pattern resembling that of chicken forelimb. COMPUCELL's modular architecture allows iteratively replacing the interactions and quantities with more detailed and realistic ones as they become available. Although our simulations use a rectangular, 2-d domain instead of a curvilinear, paddle-shaped domain, and differential equations that do not correspond exactly to the genetic processes in the living limb bud, they do adequately illustrate a general conceptual framework for vertebrate limb organogenesis. A similar statement can be made with regard to accurate measurements of tissue properties that serve as input to CPM. Figure 2 also shows growing zones and their interfaces moving in distal direction. Cells formed by division move downward (out of apical zone) and replenish active zones. Lowermost frozen zone grows as cells condense into a bonelike pattern. 


\section{Computational Model}

Activator concentration in the internal space of the limb obeys a set of reactiondiffusion PDEs (we use the Schnakenberg equations). With an appropriate choice of parameters in the Schnakenberg reaction-diffusion equations Eq. (5), which includes the space in which the reaction-diffusion mechanism operates, the activator concentration produces a sequence of arithmetically increasing numbers of vertical stripes, roughly approximating the distribution of skeletal elements in a chicken limb, see Figs. 3a-c. Cells that sense a threshold level of activator concentration locally begin to produce SAM. This is modeled as a transition from a non-SAM producing mesenchymal cell state to a cell state capable of SAM production. Thus, we have three fields of chemical concentration in our computations - for the activator, the inhibitor and SAM. COMPUCELL allows definition of an arbitrary number of fields and state transition rules of interest. In our example, the first two fields evolve according to the Schnakenberg equations, the SAM field depends on local secretion by cells and the secretion rate itself obeys an ODE. Finally, the mesenchymal cells respond to elevated SAM concentration by upregulating their cell-cell adhesion parameters, and by responding to the 'gluing' effect of SAM through the haptotaxis term in the CPM. The secretion of SAM by cells reinforces condensation of mesenchymal cells in regions which already had high SAM concentrations. We now describe the submodels.

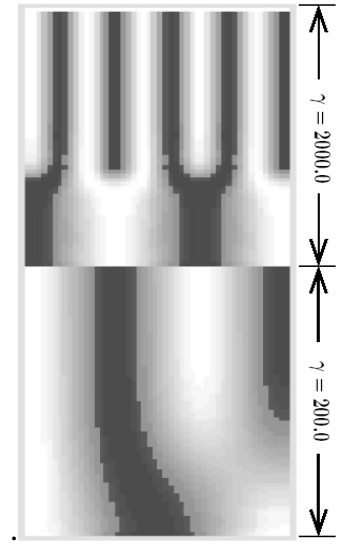

(a) $\gamma: 2$ steps

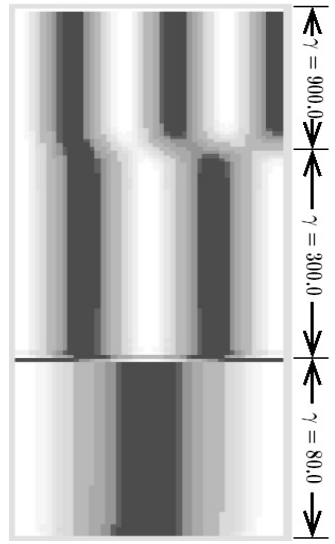

(a) $\gamma: 3$ steps

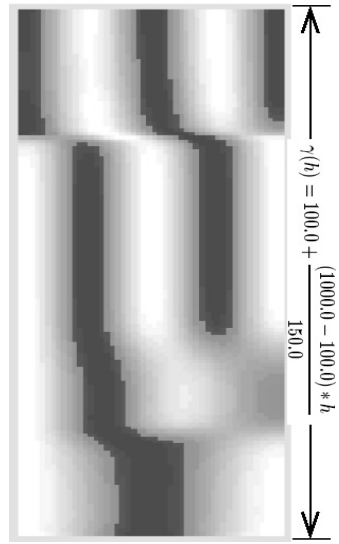

(a) $\gamma: 2$ steps

Fig. 3. Solutions of Schnakenberg equations (5) illustrating periodicity of stripes in stable solutions. Aspect ratio is 3:1. Figure is not to scale

\subsection{The Cellular Potts Model (CPM)}

CPM is an extension of the Ising model ${ }^{2}$. It can simulate condensation of cells based on differential adhesion $[9,10]$. We extend the model by (i) Adding an extra term to

\footnotetext{
${ }^{2}$ http: / /www.physics. cornell. edu/sethna/teaching/sss/ising/intro.htm
} 
model haptotaxis of cells to SAM, (ii) Allowing time variation in the adhesivity of cells, and (iii) Allowing cell growth and division

Energy Minimization Formalism: CPM dynamics use an energy minimization formalism to simulate the spatially extended cells undergoing cytoskeletally driven fluctuations. Quantitative experiments show that the CPM successfully reproduces the behavior of simple cell aggregates [15,16]. Fundamental entities in the model are individual cells. An effective energy, E, and "fields" (e.g., local concentrations of diffusants) describe cells' interactions, motion, differentiation and division. Effective energy mixes true energies, like cell-cell adhesion, and terms that mimic energies, e.g., response of a cell to a chemotactic gradient. Cells move to minimize their total effective energy. CPM superimposes a lattice on the cells, with an index (also called a spin) associated with each lattice site (pixel). Value of the index at a lattice site is $\sigma$ if the site lies in cell $\sigma$. Domains (i.e., the collection of lattice sites with the same index) in the lattice represent cells; probability that such domains are connected is high.

Cell Adhesion: The net interaction between two cell membranes is phenomenologically described by a binding energy per unit area, $J_{\tau, \tau}$, which depends on the types of the interacting cells, $\tau$ and $\tau$. Binding energy incorporates both specific (e.g., integrins, cadherins) and nonspecific interactions (e.g., elastic effects due to cell deformations, [17]). In the CPM the cell-cell interaction energy is:

$$
E_{\text {Contact }}=\sum_{(i, j, k)\left(i^{\prime}, j^{\prime}, k^{\prime}\right)} J_{\tau(\sigma), \tau^{\prime}\left(\sigma^{\prime}\right)}\left(1-\delta\left(\sigma(i, j, k), \sigma^{\prime}\left(i^{\prime}, j^{\prime}, k^{\prime}\right)\right)\right),
$$

where the Kronecker delta, $\delta\left(\sigma, \sigma^{\prime}\right)=0$ if $\sigma \neq \sigma^{\prime}$ and $\delta\left(\sigma, \sigma^{\prime}\right)=1$ if $\sigma=\sigma^{\prime}$, ensures that only surface sites between neighboring cells contribute to the cell adhesion energy. Cell adhesion molecules may change both in quantity and identity, which we can model as variations in cell-specific adhesivity.

Cell Volume, Cell Division, and Cell Death: At any time, $t$, a cell of type $\tau$ has a volume $v(\sigma, \tau)$ and surface area $s(\sigma, \tau)$. Cell volume can fluctuate around a certain (target) value, e.g., due to changes in osmotic pressure. In order to (phenomenologically) incorporate these behaviors into our model, we introduce two model parameters: volume elasticity, $\lambda$, and target volume, $v_{\text {target }}(\sigma, \tau)$. Similarly, for surface area fluctuations, we define a membrane elasticity, $\lambda$, and a target surface area, $s_{\text {tar- }}$ get $(\sigma, \tau)$. We incorporate these constraints in the energy minimization formalism of CPM by introducing energy penalties for variations in $v$ and $s$ of a cell from their target values, which we set to the average values for the particular cell types:

$$
E_{\text {volume }}=\sum_{\text {all-cells }} \lambda_{\sigma}\left(v(\sigma, \tau)-v_{\text {target }}(\sigma, \tau)\right)^{2}+\sum_{\text {all-cells }} \lambda_{\sigma}^{\prime}\left(s(\sigma, \tau)-s_{\text {target }}(\sigma, \tau)\right)^{2}
$$

We model cell growth by allowing the values of $v_{\text {target }}(\sigma, \tau)$ and $s_{\text {target }}(\sigma, \tau)$ to increase with time. Cell division occurs when the cell reaches a fixed, type-dependent volume. For another form of cell division, see [17]. We model division by starting 
with a cell of average size, $v_{\text {target }}=v_{\text {target,average; }}$ causing it to grow by gradually increasing $v_{\text {target }}$ to $2 v_{\text {target,average; }}$, and splitting the dividing cell into two, each with a new target volume: $v_{\text {target }} / 2$. One daughter cell assumes a new identity (unique value of $\sigma$ ). We can model cell death simply by setting the cell's target volume to zero.

Extracellular Matrix (ECM): We model ECM, liquid medium and solid substrates just like cells with a distinct index (spin). We must define the interaction energy between each cell type and the ECM.

Dynamics of Cell Evolution - Membrane Fluctuations: In mixtures of liquid droplets, thermal fluctuations of the droplet surfaces cause diffusion (Brownian motion) leading to energy minimization. The simplest assumption is that an effective temperature, $T$, drives cell membrane fluctuations. Fluctuations are described statistically using the Metropolis algorithm for Monte-Carlo Boltzmann dynamics; $T$ defines the size of the typical fluctuation. If a proposed change in configuration (i.e., a change in the spins associated with the pixels of the lattice) produces a change in effective energy, $\Delta E$, we accept it with probability:

$$
P(\Delta E)=1, \Delta E \leq 0 ; \quad P(\Delta E)=e^{-\Delta E / k T}, \Delta E>0
$$

where $k$ is a constant converting $T$ into units of energy.

Chemotaxis and Haptotaxis: Chemotaxis requires additional fields to describe the local concentrations $C(\vec{x})$ of the molecules in extracellular space. Equation(s) for the field depend on the particular morphogen molecule. A reaction-diffusion model is used for the activator, which triggers production of SAM by cells. Cells respond to the SAM field by 'sticking' to it. An effective chemical potential, $\mu(\sigma)$ models chemotaxis or haptotaxis. Equation 4 incorporates the effective chemical energy into the CPM energy formalism. Thus, the cells execute a biased random walk averaging in the direction of the gradient. Experiments can measure $\mu(\sigma)$.

$$
E_{\text {Chemical }}=\mu(\sigma) C(\vec{x})
$$

\subsection{Reaction-Diffusion Equations}

The Schnakenberg equations we use are the following [18]:

$$
\begin{aligned}
& \frac{\partial u}{\partial t}=\gamma\left(a-u+u^{2} v\right)+\nabla^{2} u, \\
& \frac{\partial v}{\partial t}=\gamma\left(b-u^{2} v\right)+d \nabla^{2} v .
\end{aligned}
$$

where $u$ is the activator concentration at acation $(x, y)$ at time $t ; v$ is the inhibitor concentration. $\gamma$ is a parameter that affects the period (wavelength) of the (activator) 
pattern. Solutions in Fig. 3 demonstrate how controlling $\gamma$ values can generate an activator pattern resembling chondrogenesis.

\subsection{State Transition Model}

The behavior of a cell depends on its state. State change rules depend on several chemical fields at the intra- and inter-cellular level. We assume all cells in the active zones are in a mitosing (i.e., dividing) state. When a mitosing cell in the active zones senses a threshold local concentration of activator, it enters the SAM producing state. In this state, the cell also upregulates cell-cell adhesion (the parameter $J_{\tau}, \quad\left(J_{\text {cell,cell }}\right)$ in the CPM decreases). Cells that have not experienced local threshold levels of activator are in the mitosing and condensing state; they undergo the dynamics of CPM. Such cells respond to local SAM concentration, but do not produce SAM on their own. This model of genetic regulation captures the formal, qualitative aspects of regulatory interactions and also allows fitting to quantitative experiments.

\subsection{Modeling of Zones}

For computational efficiency and biological realism, we apply the various dynamics (CPM, reaction-diffusion, state transitions) only in specific regions of growing limb bud. This zonal organization is typical of multicellular development. We thus describe zones, interfaces, and growth. The AER secretes signals that induce cell division in the proximal region; we simply assume that cell division takes place everywhere in the active zones. In the active zone, which allows for cell condensation and haptotaxis in response to SAM production, cells respond to threshold activator concentration by producing SAM and condensing into patterns governed by the activator pattern. In addition, we have an active zone for reaction-diffusion, which is slightly larger than the cells' active zone. In the reaction-diffusion active zone, activator concentration evolves to establish a pre-pattern for mesenchymal condensation. In the frozen zone, condensation into cartilaginous patterns has already occurred; no further evolution takes place here, saving on computation. In the absence of experimentally determined governing rules for these zones and their interfaces, we assume ad hoc rules for the motion of zones, based on the requirement that chemical concentration fields and cell clustering mechanisms have enough time to form distinctive patterns.

\subsection{Integration of Submodels}

We must integrate the submodels, in particular the stochastic CPM and continuum reaction-diffusion model to allow the various mechanisms to work in a coordinated fashion and simulate the full system. We must:

1. Match the spatial grid for continuum and stochastic models.

2. Define relative number of iterations for reaction-diffusion \& CPM evolvers.

The section on "Software" below describes integration in more detail. 


\section{Software}

COMPUCELL provides multiple user interfaces. A file-based input can be used to describe the parameters associated with simulation. Alternatively, the model to be simulated and the simulation can be controlled using BIOLOGO, a modeling language designed and implemented for COMPUCELL ${ }^{3}$. The front end includes: (1) A file based user interface for simulation parameters and (2) A graphical user interface for controlling the simulation and for presenting the results. Back end is split into two main engines that carry out the bulk of the number crunching: (1) A computational engine for the biological model and (2) A visualization engine for graphics.

Computational engine has three modules: (1) CPM engine (stochastic, discrete), (2) Reaction-diffusion engine (continuum, PDEs), and, (3) State transition model engine (rule based state automaton). Reaction-diffusion engine uses an explicit solver based on forward time marching. Results of these calculations are made available as objects of a 'Field' class. A Field object is also present for SAM concentration. CPM uses a field of pixels. For each of the fields an appropriate method for evolving it is used. The decision on which field to evolve is based on the criteria specified for interfacing the various grids and time scales. Grids are matched the using a simple interpolation; for time scales we specify the number of iterations for the evolution of field " 1 " before we update a field " 2 " that interacts with " 1 ". In the CPM, the spins associated with pixels evolve according to the Metropolis algorithm. "State Transition Model" is used for the evolver governing cell differentiation for the genetically determined response of the cell.

Cell Division Algorithm: A cell capable of dividing splits after growing to twice its original size. To 'grow' the cell, its target volume is gradually increased so that the target volume doubles over a predetermined number of Metropolis steps (time). Assigning a new spin to half of the cell's constituent pixels then splits the cell. A modified breadth-first search is used to select pixels to be assigned a new spin; the split is approximately along the "diameter". Visualization ToolKit (VTK), available as freeware for various operating systems from the source $\mathrm{URL}^{4}$, is used for visualization.

\section{Discussion of Simulation Results for Chicken Limb Development}

Figure 2 shows a simulation of the full model described above. Computational domain corresponds to the real anteroposterior width of $1.4 \mathrm{~mm}$; patterning begins at stage 20 of chicken embryo development. The proximodistal length of $4 \mathrm{~mm}$ at stage 28 is about three times the width. A 100x300 grid covers the domain. Cells cluster subject to differential cell adhesion. The genetically governed response of cells to high activator concentration is to begin secreting SAM. Cells respond to SAM in two ways: (1) SAM causes cells to stick to the substrate; (2) SAM makes the cells more likely to condense by upregulating cell-cell adhesion. The parameter $\gamma$ in Schnakenberg reaction-diffusion equations determines the periodicity of the pattern. Activator concentra-

\footnotetext{
${ }^{3}$ http: / / www. nd.edu/ 1cls / compucell

${ }^{4}$ http://public.kitware.com/VTK/get-software.php
} 
tion is shown at various times; formation of the pre-pattern directing later cell condensation into the chondrogenic pattern is clearly seen. Since cells exposed at some time to high activator concentration begin and continue to secrete SAM, and SAM in turn has the two effects described above, the pattern of SAM concentration resembles the activator pre-pattern. Finally, cells condense into the bone pattern of $1+2+3$ (where 3 corresponds to the three digits) of the chicken limb. Growth of the limb bud depends on cell division rate and how fast cells move. New cells generated by cell division push the limb tip upward, making the growth look more natural.

Table 1. Runtimes for different grid sizes and number of cells

\begin{tabular}{|l|l|l|l|l|l|}
\hline Grid & $\begin{array}{l}\text { Number } \\
\text { of Cells }\end{array}$ & $\begin{array}{l}\text { Cell } \\
\text { Density }\end{array}$ & $\begin{array}{l}\text { Total } \\
\text { iterations }\end{array}$ & $\begin{array}{l}\text { Time, visu- } \\
\text { alization }\end{array}$ & $\begin{array}{l}\text { Time, no } \\
\text { visualization }\end{array}$ \\
\hline $150 \times 150$ & 100 & $64 \%$ & 700 & 31 minutes & 2.5 minutes \\
\hline $300 \times 300$ & 900 & $64 \%$ & 700 & 199 minutes & 6 minutes \\
\hline $600 \times 600$ & 900 & $64 \%$ & 700 & 329 minutes & 18 minutes \\
\hline $150 \times 150$ & 325 & $52 \%$ & 700 & 34 minutes & 3.5 minutes \\
\hline
\end{tabular}

Simulations ran on a Sun-Blade-1000 with a $900 \mathrm{MHz}$ CPU and 512 Megabytes of memory. Table 1 presents data on runtimes for different grid sizes and numbers of cells. With the same grid size, the number of cells does not much affect the speed, demonstrating the scalability of our algorithms for quantities dependent on cell number. Visualizing the computed data is highly computation intensive and does not scale as well as the computation.

Acknowledgements. Support from NSF Grants IBN-0083653 and ACI-0135195 is acknowledged.

\section{References}

1. Mjolsness, E., Sharp, D. H. \& Reinitz, J. (1991) A connectionist model of development. J. Theor. Biol., 152, 429-453.

2. Davidson, E., Rast, J. P., Oliveri, P., Ransick, A., Calestani, C., Yuh, C., Minokawa, T., Amore, G., Hinman, V., C.Arenas-Mena, Otim, O., Brown, C., Livi, C., Lee, P., Revilla, R., Rust, A., Pan, Z., Schilstra, M., Clarke, P., Arnone, M., Rowen, L., Cameron, R., McClay, D., Hood, L. \& Bolouri, H. (2002) A genomic regulatory network for development. Science, 295, 1669.

3. Marée, F. M. \& Hogeweg, P. (2001) How amoeboids self-organize into a fruiting body: multicellular coordination in Dictyostelium discoideum. Proc. Natl. Acad. Sci. USA, 98, 3879-3883.

4. Newman, S. A. \& Comper, W. (1990) Generic physical mechanisms of morphogenesis and pattern formation. Development, 110, 1-18.

5. Lewis, J. (1975) Fate maps and the pattern of cell division: a calculation for the chick wing-bud. J. Embryol. exp. Morph., 33 , 419-434.

6. Newman, S. A. (1996) Sticky fingers: Hox genes and cell adhesion in vertebrate limb development. BioEssays, 18, 171-174. 
7. Downie, S. \& Newman, S. (1995) Different roles for fibronectin in the generation of fore and hind limb precartilage condensations. Dev. Biol., 172, 519-530.

8. Oberlender, S. \& Tuan, R. (1994) Expression and functional involvement of N-cadherin in embryonic limb chondrogenesis. Development, 120, 177-187.

9. Steinberg, M. S. (1978). Specific cell ligands and the differential adhesion hypothesis: How do they fit together? In Specificity of Embryological Interactions (D. R. Garrod, Ed.), pp. 97-130. London: Chapman and Hall.

10. Graner, F. \& Glazier, J. A. (1992) Simulation of biological cell sorting using a twodimensional extended Potts model. Phys. Rev. Lett., 69, 2013-2016.

11. Jong, H. D. (2002) Modeling and simulation of genetic regulatory systems: a literature review. J. Comp. Biol., 9, 67-103.

12. Turing, A. (1952) The chemical basis of morphogenesis. Phil. Trans. Roy. Soc. London, B 237, 37-72.

13. Newman, S. A. \& Frisch, H. L. (1979) Dynamics of skeletal pattern formation in developing chick limb. Science, 205, 662-668.

14. Meinhardt, H., and Gierer, A. (2000) Pattern formation by local self-activation and lateral inhibition. Bioessays 22, 753-760.

15. Mombach, J. \& Glazier, J. (1996) Single cell motion in aggregates of embryonic cells. Phys. Rev. Lett., 76, 3032-3035.

16. Marée, S. (2000) From Pattern Formation to Morphogenesis. PhD thesis, Utrecht University Netherlands.

17. Drasdo, D. \& Forgacs, G. (2000) Interplay of generic and genetic mechanisms in early development. Developmental Dynamics, 219, 182-191.

18. Murray, J. D. (1993) Mathematical Biology, Second Corrected Edition (Biomathematics Vol. 19), Springer-Verlag, Berlin Heidelberg, pp. 156, 376, 406, 472, 739. 